BNL-95295-2011-IR

\title{
Testing of Kaonetics' Devices at BNL
}

\author{
A. Bolotnikov, G. Smith and R. James
}

June 2011

\author{
Nonproliferation and National Security Department \\ Brookhaven National Laboratory
}

\section{U.S. Department of Energy \\ National Nuclear Security Administration, Office of Science}

\footnotetext{
Notice: This manuscript has been authored by employees of Brookhaven Science Associates, LLC under Contract No. DE-AC02-98CH10886 with the U.S. Department of Energy. The publisher by accepting the manuscript for publication acknowledges that the United States Government retains a non-exclusive, paid-up, irrevocable, world-wide license to publish or reproduce the published form of this manuscript, or allow others to do so, for United States Government purposes.
} 


\section{DISCLAIMER}

This report was prepared as an account of work sponsored by an agency of the United States Government. Neither the United States Government nor any agency thereof, nor any of their employees, nor any of their contractors, subcontractors, or their employees, makes any warranty, express or implied, or assumes any legal liability or responsibility for the accuracy, completeness, or any third party's use or the results of such use of any information, apparatus, product, or process disclosed, or represents that its use would not infringe privately owned rights. Reference herein to any specific commercial product, process, or service by trade name, trademark, manufacturer, or otherwise, does not necessarily constitute or imply its endorsement, recommendation, or favoring by the United States Government or any agency thereof or its contractors or subcontractors. The views and opinions of authors expressed herein do not necessarily state or reflect those of the United States Government or any agency thereof. 


\section{Testing of Kaonetics’ Devices at BNL}

\section{A. Bolotnikov, G. Smith, and R. James}

The goal of these measurements was to evaluate whether there is evidence of emission of X-rays, gamma-rays, and neutrons by devices developed by Kaonetics Technologies, Inc. during their operation. The measurements were carried out with two Kaonetics's modules, called EMR Gen 1 ("Device 1") and EMR Gen 2 ("Device 2"). Two types of detectors were set up in a remote building to avoid potential interference from other labs. For X- and gamma-rays we used a large-volume, high-sensitivity NaI(Tl) Bicron scintillation detector and a multichannel analyzer (MCA). For neutrons, we employed a $\sim 1 / 2$ liter ${ }^{3} \mathrm{He}$-filled proportional counter. The measurements were taken under normal background-dominated conditions.

As described in the summaries below, we observed no statistically significant emissions of X-rays, gamma-rays or neutrons during the operation of these devices.

\section{1. $\mathrm{X}$ - and gamma-ray measurements}

The NaI detector was placed $\sim 2$ feet from the emitting horns of the device under test. The detector was connected via long cables to electronics located far away from the device to avoid electromagnetic interference. The detector was calibrated with a standard

${ }^{137} \mathrm{Cs}$ source and backgrounds were measured before and after every test. In addition, to improve the sensitivity, the backgrounds were measured over night. The pulse-height spectra were collected for $~ 5$ min when the devices were in the "On" and "Off" states. The low- and high-threshold of energy limits in these measurements were $10 \mathrm{keV}$ and 2.4 MeV, respectively. The measurements were taken for two energy ranges corresponding to the low and high gains of the amplifier. The low-gain range was used primarily to detect $\mathrm{X}$ and low-energy gamma rays, while the high-gain range was used to detect the gamma ray up to $2.4 \mathrm{MeV}$. No statistically significant counts from the devices were detected above background in 5-min exposure times.

\subsection{High-gain measurements}

Fig. 1 shows the calibration spectrum measured from a $10-\mu \mathrm{Ci}{ }^{137} \mathrm{Cs}$ source. Both $38 \mathrm{keV}$ X-ray and $662 \mathrm{keV}$ gamma-ray lines are seen in the pulse-height spectrum. Fig. 2 shows the normalized background spectra measured for $18 \mathrm{~h}$ (red) and $\sim 5$ min (black) immediately after the device was shut down. The spectrum measured when operating Device 1 ("On” state) for $\sim 5$ min is shown in Fig. 3, together with the background spectrum normalized (scaled) to the same acquisition time (live time). No statistically significant X-or gamma-rays were detected over the background.

\subsection{Low-gain measurements}

In a similar manner to the low-gain measurements, Figs. 4 and 5 show the background spectra and the spectrum accumulated during the test of Device 1. Fig. 6 shows the 
spectrum measured from Device 2 in $~ 3$ min. For comparison, the background spectrum measured immediately after Device 2 was powered down is shown in Fig. 7.

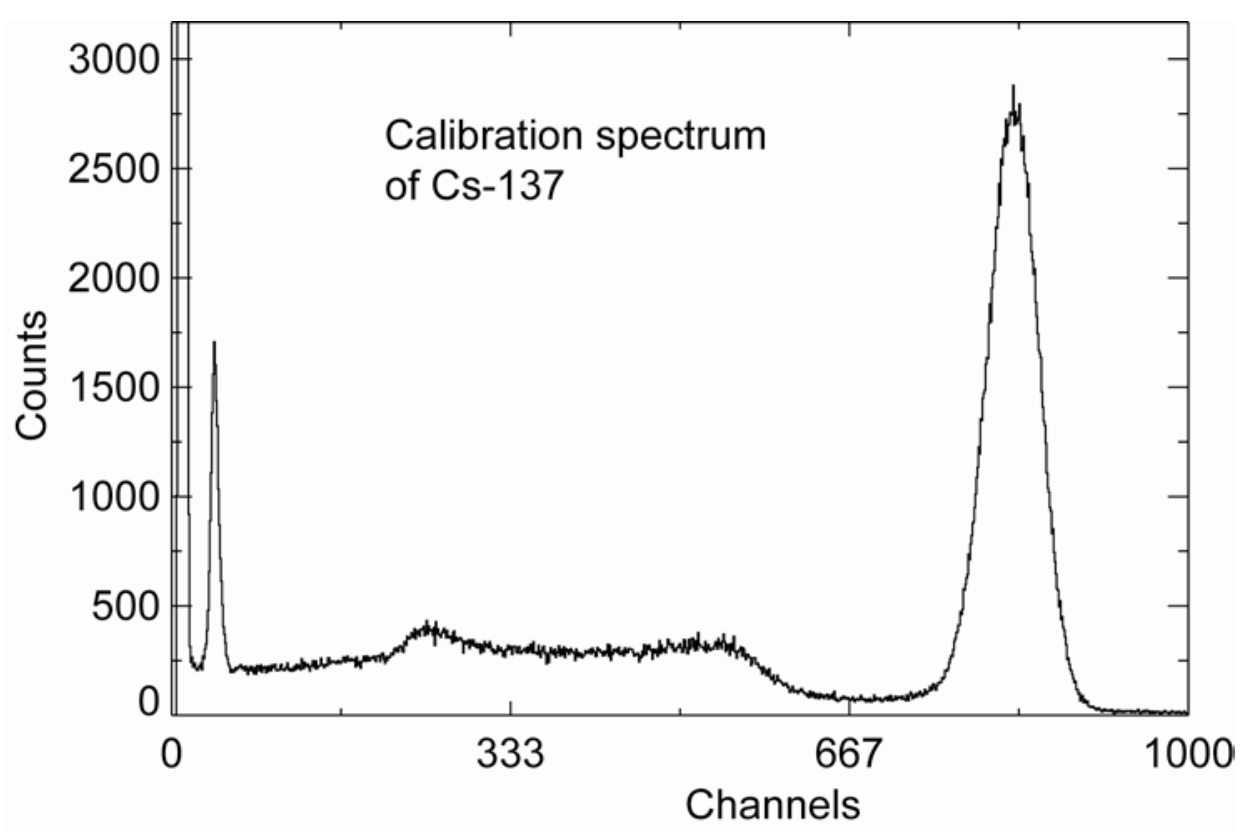

Fig. $1 .{ }^{137}$ Cs spectrum used to calibrate the spectroscopy electronics.

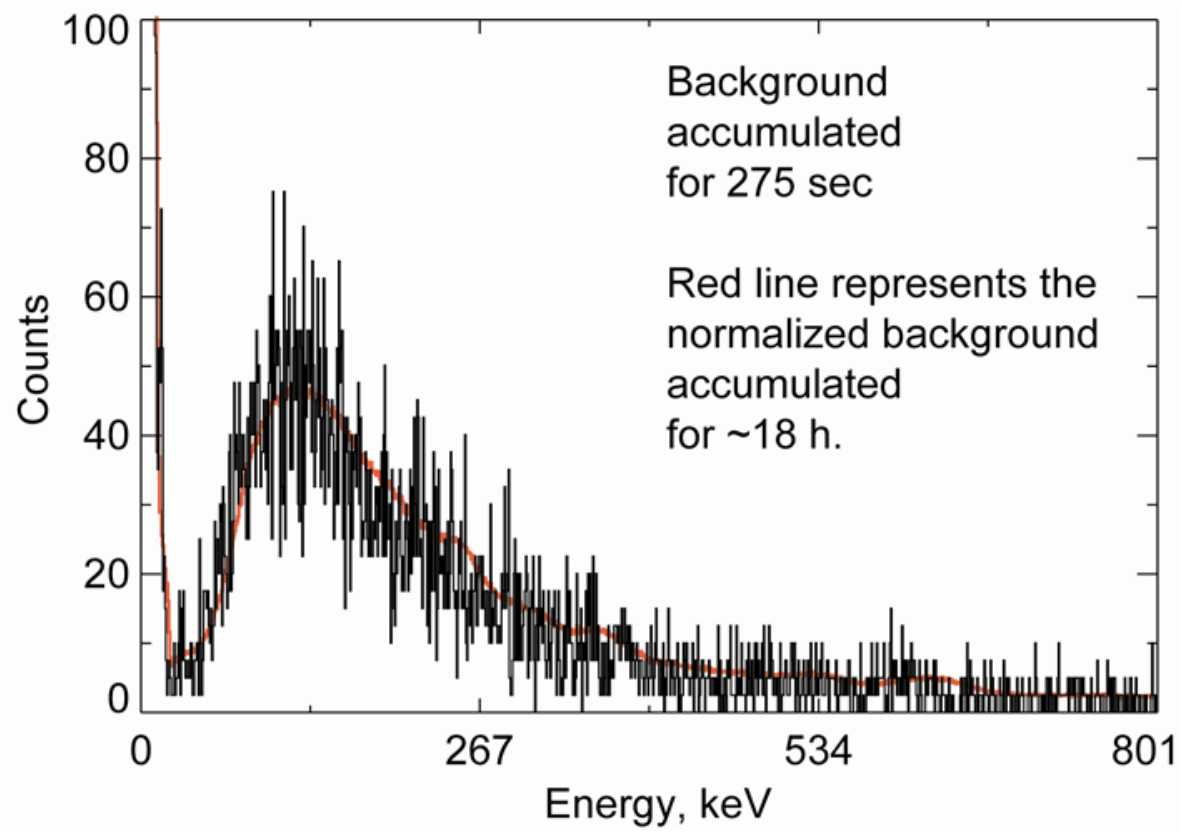

Fig. 2. Normalized background spectra measured for $\sim 18 \mathrm{~h}$ (red) and in $\sim 5$ min (black) immediately after the device was shut down. 


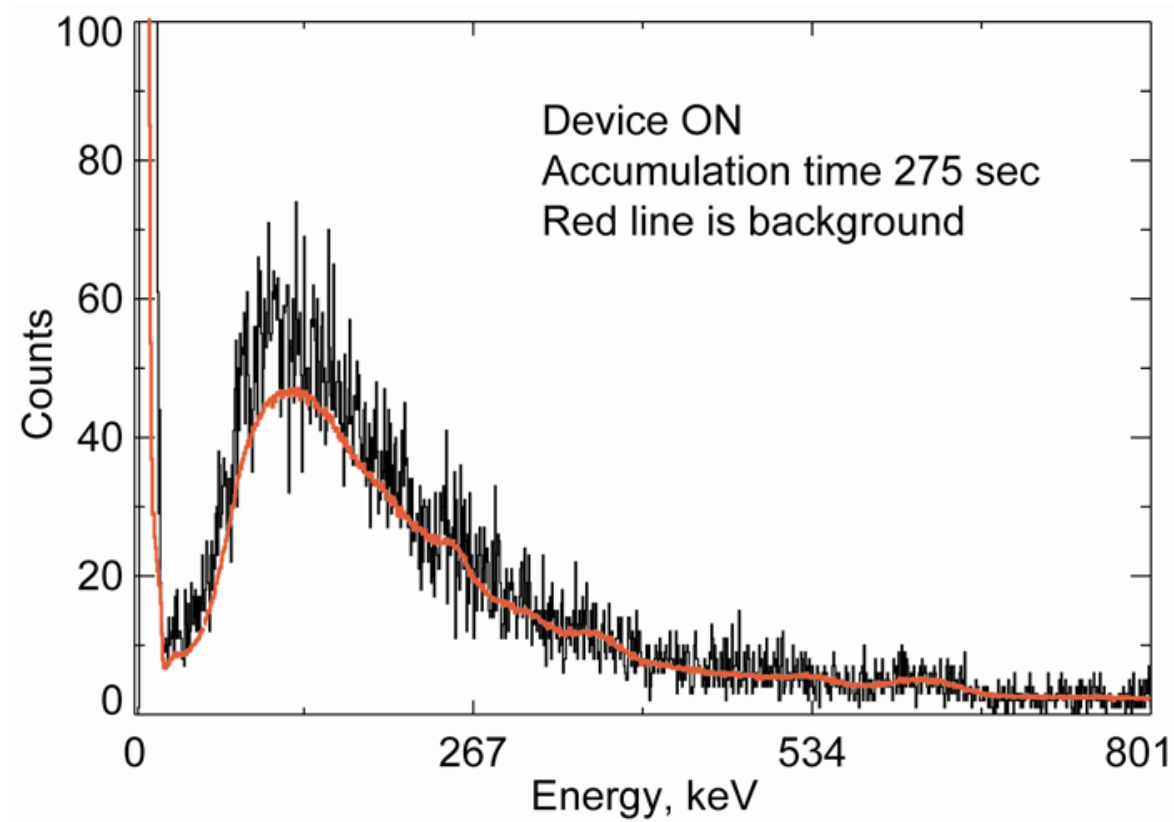

Fig. 3. Spectrum measured when operating Device 1 (“On” state) in 5 min (black). The background spectrum normalized (scaled) to the same acquisition time (live time) is also shown (red). No statistically significant $\mathrm{X}$ - or gamma rays were detected above the background.

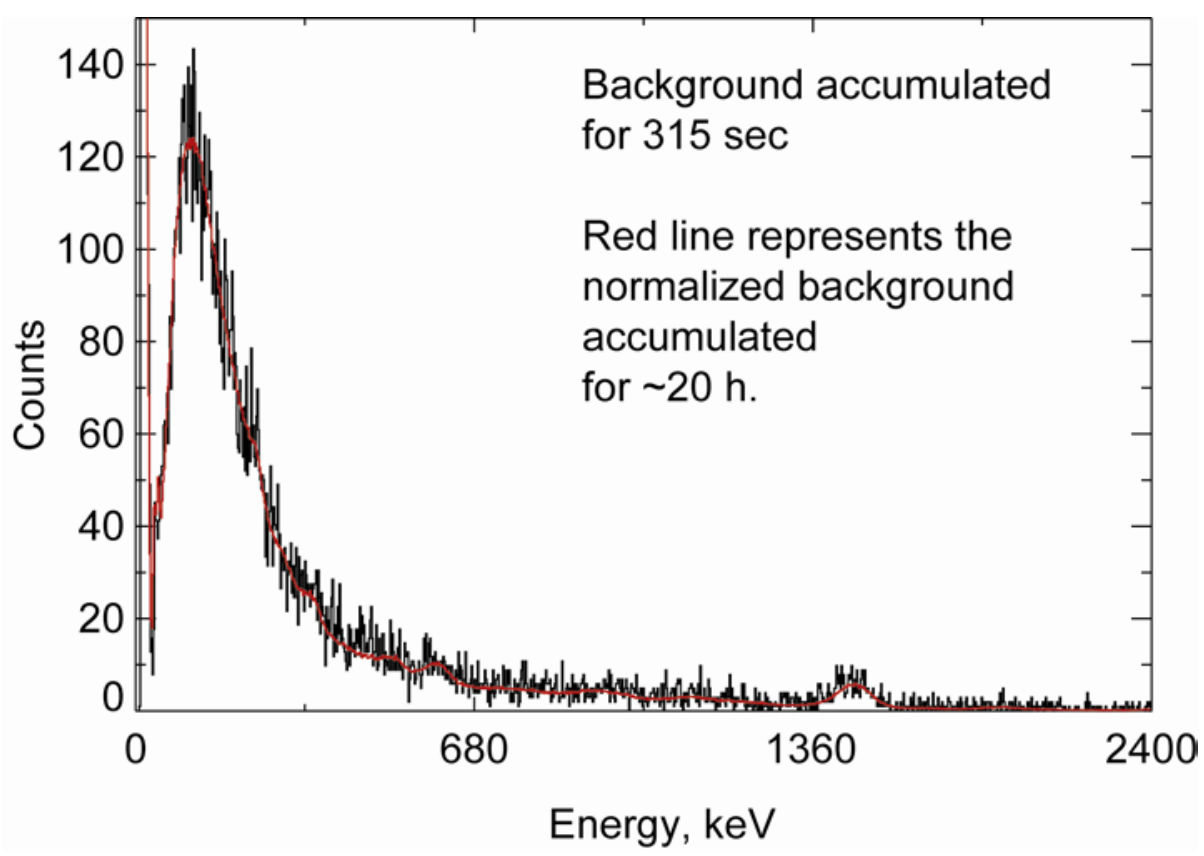

Fig. 4. Normalized background spectra measured at high gain for $\sim 20 \mathrm{~h}$ (red) and in $\sim 5$ min (black) immediately after the device was powered down. 


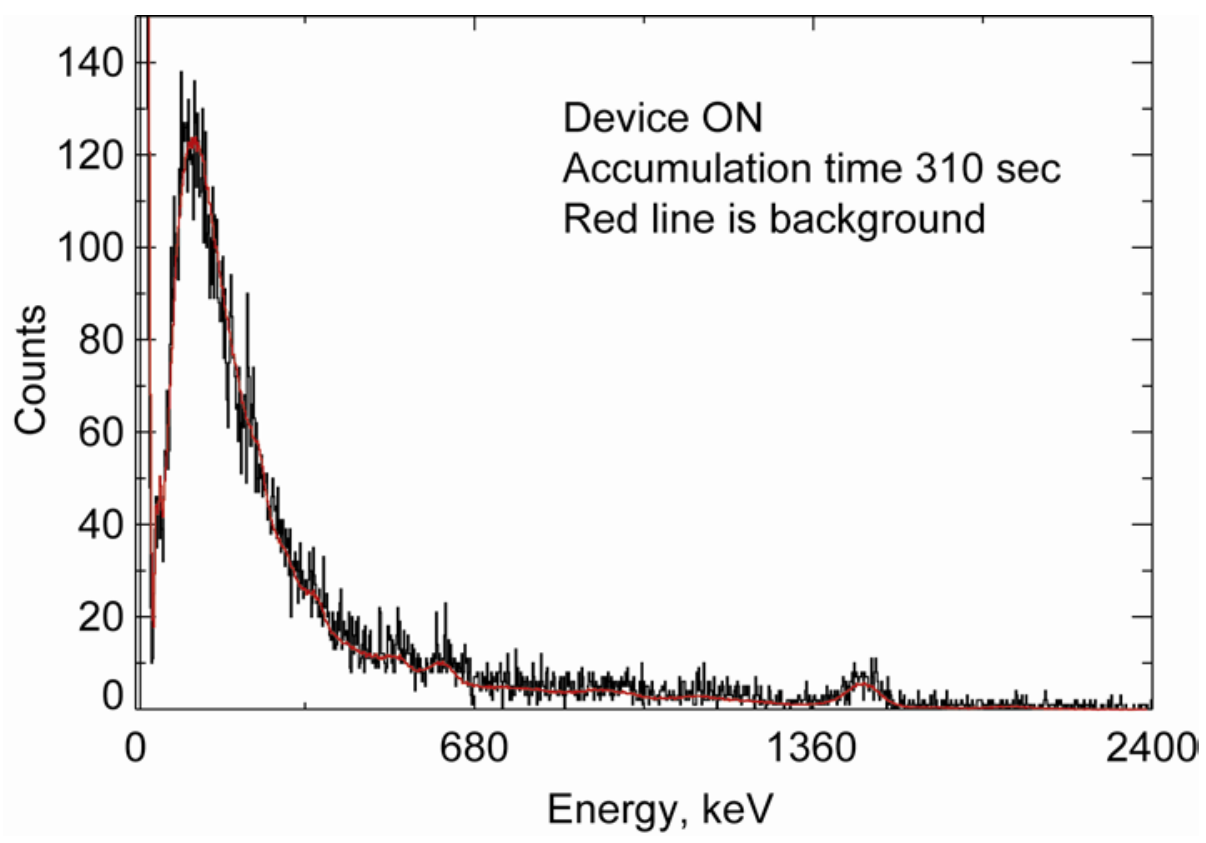

Fig. 5. Spectrum measured when operating Device 1 (“On” state) at low gain in 5 min (black). The background spectrum normalized (scaled) to the same acquisition time (live time) is also shown (red). No statistically significant counts were detected above background.

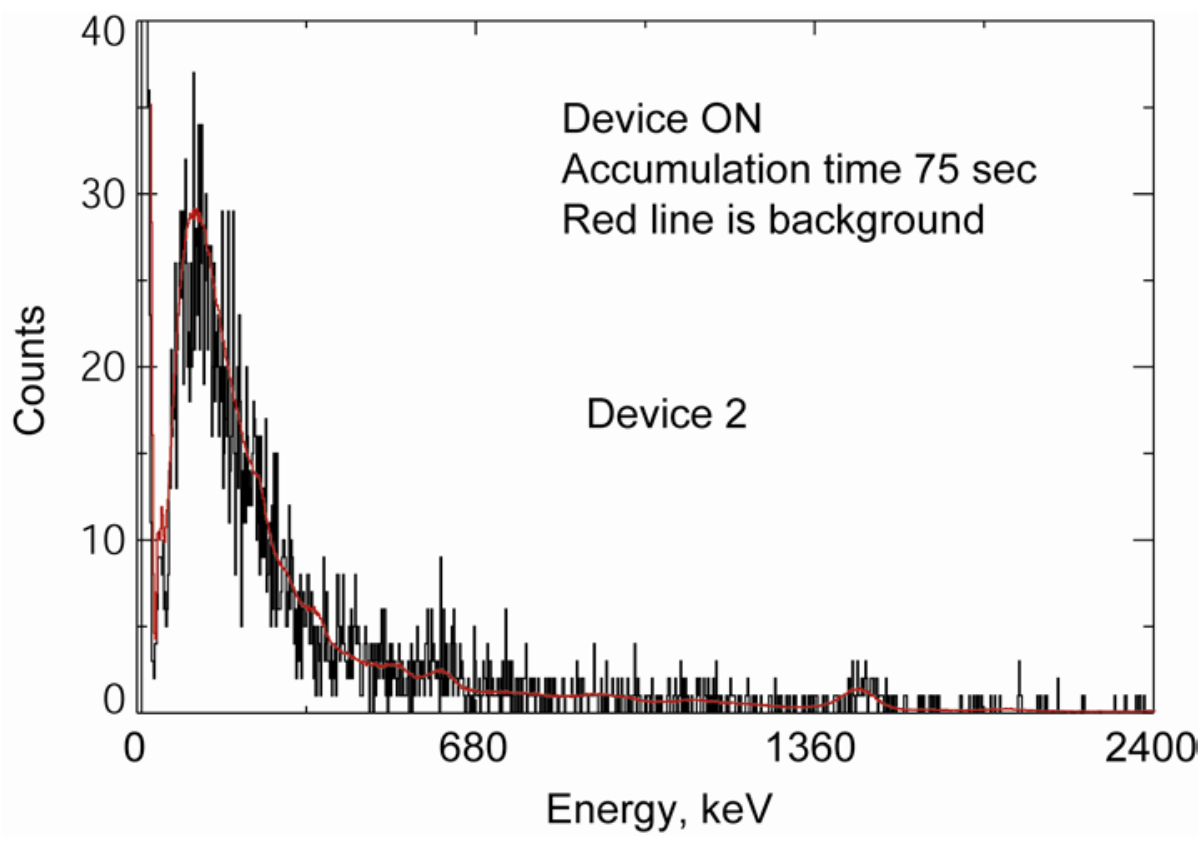

Fig. 6. Spectrum measured when operating Device 2 (“On” state) in 3 min (black). The background spectrum normalized (scaled) to the same acquisition time (live time) is also shown (red). No statistically significant $X$ - or gamma rays were detected above the background. 


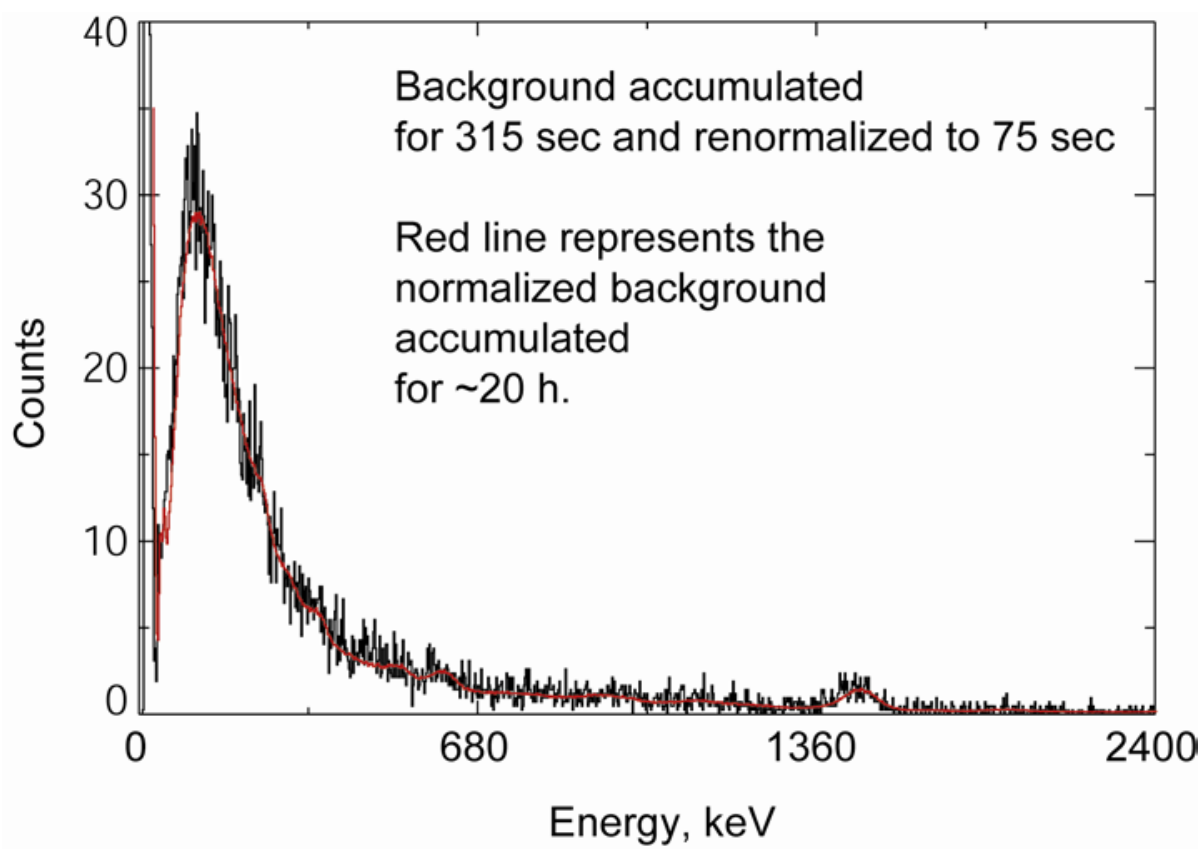

Fig. 7. Normalized (scaled) to 75 sec background spectra measured at high gain for $\sim 20 \mathrm{~h}$ (red) and for $\sim 5$ min (black) immediately after the device was powered down (scaled to $75 \mathrm{sec})$.

\subsection{Test for potential secondary X-rays generated by Device 1 in a $7 \mathrm{~mm}$ thick tungsten plate}

a) X-ray measurements

This test was conducted to verify the hypothesis that the radiation emitted by Device1 can potentially generate secondary X-rays in a tungsten plate. Fig. 8. shows the pulse-height spectra measured with the NaI-detector operating in high-gain mode with and without the tungsten plate between Device 1 and the detector.

b) Neutron measurements

Because of strong interference, the neutron detector was positioned $\sim 5$ feet away from Device 1. Using an oscilloscope we visually evaluated any increase above background of the count rate generated by neutrons from Device 1 in the operating state ("On”). Typical background (cosmic neutrons) was $\sim 1$ event per $10 \mathrm{sec}$. No increase in the rate of neutron events was observed. 


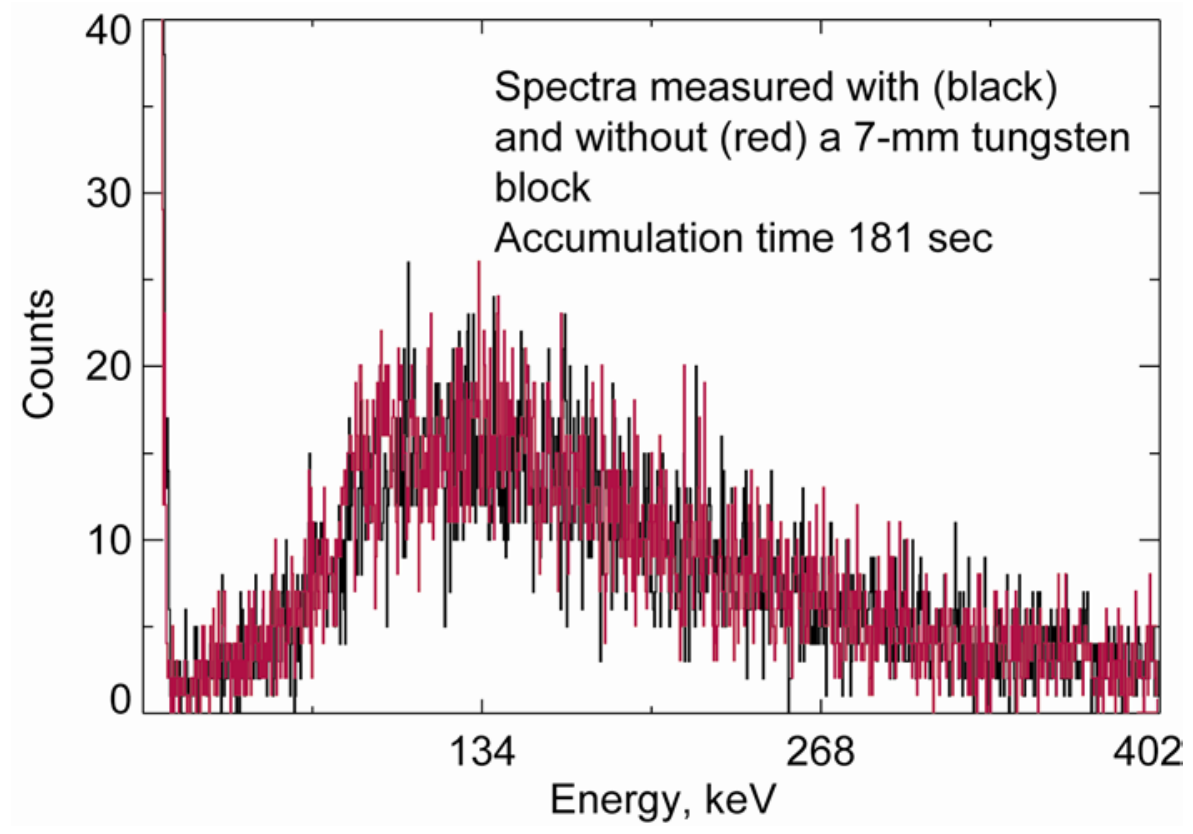

Fig. 8. Pulse-height spectra measured with the NaI-detector operating in high-gain mode with (red) and without (black) the tungsten plate placed between Device 1 and the detector. No extra counts due to potential secondary $X$-rays were observed above the background. 